\title{
Hemivértebra em cães - Revisão de literatura
}

\section{Hemivertebrae in dogs - Literature review}

\section{Resumo}

Hemivértebras são anomalias congênitas encontradas mais frequentemente em cães braquicefálicos com cauda helicoidal. Representam em torno de $7 \%$ das alterações em coluna relatadas em literatura. Ocorrem mais comumente na região da coluna tóraco-lombar, causando sintomas de lesão de neurônio motor superior em membros pélvicos. Os cães podem apresentar sintomatologia desde filhotes com progressão durante o crescimento. O diagnóstico é efetuado com base nos sintomas, exame físico e exames de imagem. O tratamento pode ser conservativo em animais sem progressão dos sintomas, porém, em animais com a progressão destes, o mais indicado, atualmente, é a estabilização cirúrgica, com ou sem descompressão medular.

\section{Summary}

Hemivertebrae are congenital anomalies found most often in brachiocephalic dogs with helical tails. These anomalies represent around $7 \%$ of the spinal alterations reported in the literature. They occur most commonly in the thoracolumbar region of the spine, causing symptoms of upper motor neuron damage in hind limbs. Dogs may display symptoms early in life which progress during growth. Diagnosis is based on symptoms, physical examination, and imaging studies. Treatment may be conservative in animals whose symptoms do not progress, but in animals whose symptoms progress, the treatment currently most indicated is surgical stabilization, with or without spinal decompression. 
Universidade de Guarulhos

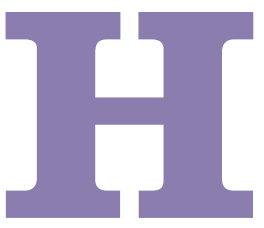

emivértebras são falhas na formação adequada do corpo vertebral. Encontradas com maior frequência em cães braquicefálicos com cauda helicoidal (DEWEY; COATES, 2007), como o Buldogue Inglês, o Buldogue Francês, o Boston Terrier e o Pug, já que a cauda helicoidal ocorre devido a uma hemivértebra coccígea (DONE et al., 1975 apud JEFERRY; SMITH, TALBOT, 2007). Geralmente, são achados de exame (CHARALAMBOUS et al., 2014) a sua ocorrência dentre alterações em coluna vertebral é de 7,74\% (SANTOS et al., 2006), sendo a de maior prevalência entre as alterações congênitas (SCHLENSKER; DISTL, 2013). Schlensker e Distl (2013) relataram hemivértebra em 86,7\% dos 105 Buldogues Franceses examinados.

A hemivértebra ocorre por defeito na formação do corpo vertebral e não por falha na segmentação do mesmo (VAN SCHIRK, 1932 apud McMASTER; SINGH, 1999). A hemivértebra desenvolve-se nos estágios tardios de condrificação e ossificação embrionária devido a falha na vascularização local, quando a anatomia de medula espinhal já está definida, não havendo anomalias na medula (TSOU et al., 1980 apud McMASTER; SINGH, 1999).

A hemivértebra pode ser causada por quatro tipos de defeitos, aplasia ventral e unilateral, aplasia ventral, aplasia ventral e medial, e hipoplasia ventral (McMASTER; SINGH, 1999). As vértebras com aplasia ventral e unilateral e as vértebras com aplasia ventral são mais instáveis tendendo a extruir dorsalmente, causando compressão ventral da medula espinhal (McMASTER; SINGH, 1999). Anomalias ventrais causam cifose, ao passo que anomalias unilaterais causam escoliose. 
Cães podem apresentar uma única hemivértebra ou múltiplas, que podem ser sequenciais ou distribuídas pela coluna vertebral (MOISSONNIER; GOSSOT; SCOTTI, 2011). Moissonnier, Gossot e Scotti (2011) na Escola Nacional de Veterinária de Alfort relataram que a hemivértebra única foi a manifestação mais comum, acometendo $57 \%$ dos cães por eles atendidos, seguido por hemivértebras múltiplas sequenciais, $31 \%$ dos cães acometidos, porém Besalti et al. (2005) na Universidade de Ankara, relataram que quatro dos cinco casos por ele analisados apresentavam hemivértebras múltiplas.

O aparecimento de sinais clínicos em cães com hemivértebra é raro, mas quando ocorrem podem ser graves (MOISSONNIER; GOSSOT; SCOTTI, 2011). Geralmente, devido à compressão medular, os cães afetados apresentam sinais desde filhotes que progridem, porém, os sinais também podem surgir durante o crescimento do animal, devido à instabilidade entre hemivértebra e vértebra adjacente causando compressão progressiva e crônica (McMASTER; SINGH, 1999; AIKAWA et al., 2007; MOISSONNIER; GOSSOT; SCOTTI, 2011), ou após trauma agudo ou trações de coluna (AIKAWA et al., 2007). A instabilidade vertebral da região ocorre devido ao comprometimento de processos articulares e conformação vertebral (COSTA; SAMII, 2010).

Os sinais clínicos correspondem à parte anatômica afetada pela anomalia e podem ser desde parapesia ambulatória até paraplegia, esses sinais podem ser agudos ou progressivos (AIKAWA et al., 2007). As vértebras mais comumente acometidas são as incluídas em região T3-L3 (JEFERRY; SMITH; TALBOT, 2007) e determinam sinais típicos de lesão de neurônio motor superior em membros pélvicos, tais como: incoordenação, perda de propriocepção, reações posturais retardadas, reflexos patelar e isquiático normais a aumentados. À palpação epaxial da coluna pode haver sensibilidade dolorosa e o panículo pode estar ausente na região afetada (TAYLOR, 2010).

$O$ ângulo de cifose causado pela hemivértebra determina os sinais clínicos e pode ser determinado por radiografias convencionais (MOISSONNIER; GOSSOT; SCOTTI, 2011).

\section{Outros achados}

Schey (1976) relatou a associação de má-formação vertebral congênita, entre elas a hemivértebra, com anormalidades somatoviscerais em humanos, como alterações em válvulas cardíacas, em grandes vasos, alteração morfológica de rins, ânus imperfurado, entre outras, porém em cães, tais relatos são raros.

Grenn e Lindo (1969) relataram o caso de um Pug com cifose e escoliose torácica severa devido a múltiplas hemivértebras torácicas associadas a deformidades em costelas. As costelas do lado direito apresentavam fusão e comprimento anormal com corpo delgado, enquanto no lado esquerdo apresentavam-se curtas e grossas formando uma corcunda na linha dorsal lateral esquerda do tórax. Devido a estas alterações o animal apresentava coração comprimido em seu eixo longitudinal e forçado para o hemitórax direito. Ao exame radiográfico, o diafragma não possuía seu formato característico e os lobos pulmonares não estavam em sua posição topográfica habitual.

Jakowski (1977) da Universidade de Connecticut relatou o caso de um filhote de Labrador com hemivértebra torácica e cólon duplicado, Carvallo, Domínguez e Morales (2010) da Universidade de Santo Tómas relataram a ocorrência de uma cadela sem raça definida com hemivértebra lombar e ectrodactilia bilateral, mas, em ambos os relatos, as anomalias não foram relacionadas com uma causa em comum. Berlanda et al. (2011) da Universidade de Pádua relataram o caso de um Flat Coated Retriever, macho, de três meses, com hemivértebra torácica associada a hérnia diafragmática peritônio-pericárdica. No entanto, como a sua mãe recebeu amoxicilina durante a terceira semana de prenhez, não conseguiram determinar se a hérnia diafragmática peritônio-pericárdica foi determinada pela medicação ou se teve origem genética.

\section{Diagnóstico}

O diagnóstico da hemivértebra deve ser realizado pela anamnese, exame físico e exames de imagem. O teste do panículo e a palpação da coluna vertebral auxiliam na localização da lesão (DONE et al., 1975 apud JEFERRY; SMITH; TALBOT, 2007).

Imagens radiográficas de hemivértebra geralmente não mostram estenose do canal vertebral ou compressão medular dinâmica, somente alteração na angulação vertebral (AIKAWA et al., 2007; MOISSONNIER; GOSSOT; SCOTTI, 2011), mas isso não significa que tais alterações não ocorram (BERLANDA et al., 2011). Exames mais detalhados como a mielografia evidenciam estenose do canal espinhal (MOISSONNIER; GOSSOT; SCOTTI, 2011). A mielografia sob estresse pode detectar compressão medular dinâmica e instabilidade vertebral, mas deve ser realizada com cuidado para que não ocorram danos à medula espinhal (AIKAWA et al., 2007).

A localização da hemivértebra pelo exame físico ou exame radiográfico prévios é importante para reduzir o tempo de exame de ressonância magnética ou tomografia computadorizada (COSTA; SAMII, 2010), exames necessários para o diagnóstico preciso (BERLANDA et al., 2011) e planejamento cirúrgico da hemivértebra. A 
maior vantagem da tomografia computadorizada em relação às radiografias convencionais é a melhor resolução espacial, sendo a técnica de imagem mais adequada para partes ósseas. A ressonância magnética é a técnica de escolha para tecidos moles, como medula espinhal, nervos e discos intervertebrais. Apesar de a mielotomografia ser ainda muito utilizada, ela não é necessária, pois a ressonância magnética pode alterar o contraste dos tecidos com a aplicação de sequências de aquisições diferentes, evitando o risco de morbidade de injeção de contraste no espaço epidural. Sendo assim, deve-se optar pela tomografia computadorizada quando uma estabilização vertebral cirúrgica está sendo planejada ou realizar uma ressonância magnética em animais com déficits neurológicos para determinar a compressão medular (COSTA; SAMII, 2010).
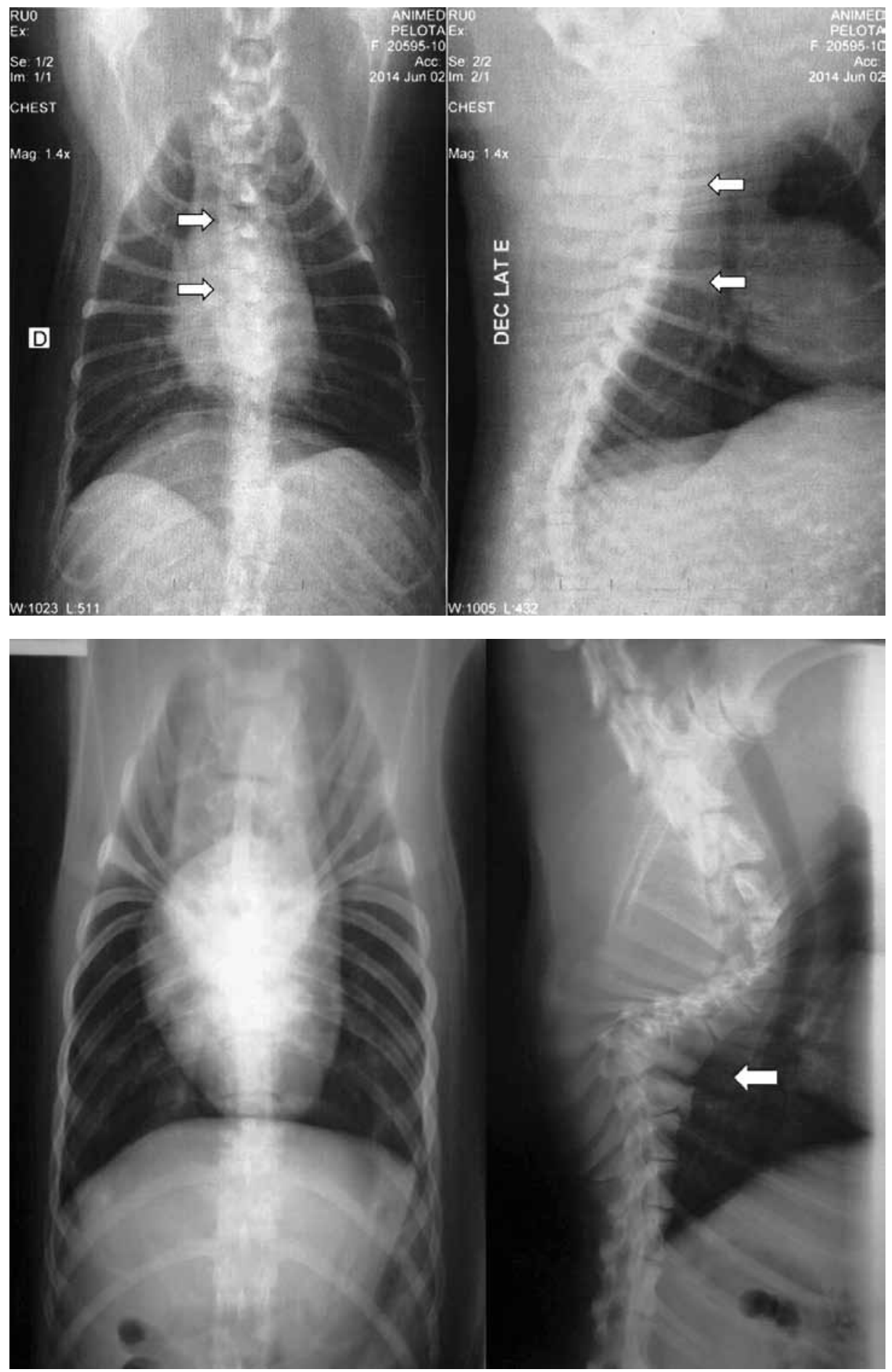

Figura 1 - Hemivértebras (indicadas pelas setas) em um Buldogue Francês, fêmea de seis meses. Apresentava algia aguda.

Fonte: Pinatti, 2014
Figura 2 - Hemivértebras (indicadas pela seta) em um canino sem raça definida, macho de nove meses. Apresentava perda de propriocepção em membros pélvicos.

Fonte: Alves e Segala, 2015 


\section{Tratamento}

O tratamento pode ser conservativo nos casos de não progressão da doença, que, geralmente, se estabiliza com o término do crescimento do esqueleto, porém, em casos de sintomas progressivos, a intervenção cirúrgica é indicada (JEFFERY; SMITH; TALBOT, 2007). O objetivo do tratamento cirúrgico deve ser a estabilização da coluna vertebral, com ou sem descompressão medular e não a correção da angulação de coluna vertebral. A descompressão convencional sem estabilização vertebral pode desestabilizar a hemivértebra, causando subluxação vertebral (AIKAWA et al., 2007). Em humanos, o manejo de pacientes com cifose congênita é feito, preferencialmente, em indivíduos jovens, com até cinco anos de idade (McMASTER; SINGH, 1999), evitando-se, assim, a progressão de sintomas com o crescimento do indivíduo.

O tratamento da cifose causado pela hemivértebra pode ser realizado com a técnica de realinhamento e estabilização com ou sem abertura do canal vertebral (MOISSONNIER; GOSSOT; SCOTTI, 2011). O uso da laminectomia permite que o cirurgião coloque o pino corretamente, sem lesar a medula, com maior facilidade, já que a medula espinhal pode ser visualizada (AIKAWA et al., 2007). A colocação de pinos percutâneos pode ser guiada por fluoroscópio, o que reduz a dissecação tecidual, o risco de lesão medular e a morbidade pósoperatória (WHEELER et al., 2007).

Em casos de compressão medular dinâmica, todo o segmento que contribui para a instabilidade vertebral deve ser estabilizado (AIKAWA et al., 2007).

Para a estabilização vertebral com fixação rígida, deve-se realizar a colocação de pinos no corpo vertebral. Em alguns casos, os pinos são colocados no processo transverso: nesse método, uma quantidade maior de pinos é necessária para a estabilização adequada (AIKAWA et al., 2007). A fixação externa do esqueleto também pode ser utilizada com a finalidade de realinhamento da coluna vertebral. Para isso, os pinos devem ser passados no corpo vertebral a $30^{\circ}$ da horizontal, sendo dois pinos em cada corpo vertebral, um na metáfise cranial e outro na metáfise caudal. Todos os pinos ipsolaterais devem estar em metáfises correspondentes, ou seja, todos os pinos de um mesmo lado devem estar nas metáfises craniais, enquanto os contra-laterais devem estar nas metáfises caudais, garantindo a simetria do fixador externo (WHEELER et al., 2007).

$\mathrm{O}$ uso de pinos com perfil de rosca positivo apresenta vantagens em relação aos pinos lisos ou perfil de rosca negativo. Pinos com perfil de rosca positivo possuem força pull-out maior, necessitando de menor quantidade de parafusos. Também, após perfurar a cortical proximal do corpo vertebral, o pino pode avançar com o drill em baixa rotação, permitindo uma inserção mais segura. $\mathrm{O}$ pino com perfil de rosca positivo tem menor chance de quebra em sua região de rosca que o pino com perfil de rosca negativo (AIKAWA et al., 2007).

Aikawa et al. (2007) relataram que oito de seus nove pacientes recuperaram a capacidade ambulatória após tratamento cirúrgico, enquanto Jeffery et al. (2007) relataram melhora do quadro de ataxia em um de seus três casos. Charalambous et al. (2014) relataram melhora da ataxia em nove cães e resolução da incontinência fecal e urinária em todos os cinco cães que apresentavam tais alterações.

\section{Conclusão}

A hemivértebra é uma anomalia vertebral congênita com implicações na qualidade de vida dos cães, que pode ser diagnosticada por um simples exame físico e auxílio de radiografias. Exames de imagem determinam se a sintomatologia apresentada é devida à angulação da coluna vertebral, à estenose do canal vertebral ou a ambas. A tomografia computadorizada ou a ressonância magnética é necessária para o planejamento cirúrgico e determinação de prognóstico.

O tratamento atual baseia-se na estabilização da coluna vertebral, evitando lesões crônicas ou compressão dinâmica da medula espinhal e descompressão medular, realizada em casos de estenose do canal vertebral. O tratamento cirúrgico leva à melhora na qualidade de vida dos animais.

\section{Agradecimentos}

Agradeço à médica veterinária Nicole Marino Pinatti e aos professores da clínica escola UnG, Alexandre Speri Alves e Renato Dalcin Segala pelas imagens cedidas. 


\section{Referências}

AIKAWA, T.; KANAZONO, S.; YOSHIGAE, Y.; SHARP, N. J. H.; MUÑANA, K. R. Vertebral stabilization using positively threaded profile pins and polymethylmethacrylate, with or without laminectomy, for spinal canal stenosis and vertebral instability caused by congenital thoracic vertebral anomalies. Vet. Surg., v. 36, n. 5, p. 432-441, 2007.

BERLANDA, M.; ZOTTI, A.; BRANDAZZA, G.; POSER, H., CALÓ, P.; BERNARDINI, M. Magnetic resonance and computed tomographic features of 4 cases of canine congenital thoracic vertebral anomalies. Can. Vet. J., v. 52, n. 12, p. 1334-1338; 2011.

BESALTI, O.; AHMET, O.;PEKCAN, Z., EMINAGA, S. Nasca classification of hemivertebra in Five dogs. Ir. Vet. J., v. 58, n. 12, p. 688-690, 2005.

CARVALLO, F. R.; DOMÍNGUEZ, A. D.; MORALES, P. C. Bilateral ectrodactyly and spinal deformation in a mixed-breed dog. Can. Vet. J., v. 51, p. 47-49, 2010.

COSTA, R. C.; SAMII, V. F. Advanced imaging of the spine in small animal. Vet. Clin. North Am. Small Anim. Pract., v. 40, n. 5, p. 765-790, 2010.

CHARALAMBOUS, M., JEFFERY, N. D., SMITH, P. M., GONÇALVES, T., BARKER, A., HAYES, G., IVES, E., VANHAESEBROUCK, A. E. Surgical treatment of dorsal hemivertebrae associated with kyphosis by spinal segmental stabilisation, with or without decompression. Vet. J., v. 202, n. 2, p. 267-273, 2014

DEWEY, C. W.; COATES, J. R. Condições diversas da medula espinhal e lesões dos nervos periféricos: distúrbios mistos da medula espinhal. In: SLATTER, D. Manual de cirurgia de pequenos animais. 3. ed. Barueri: Manole, 2007, v. 2, cap. 81, p. 1211.

JAKOWSKI, R. M. Duplication of colon in a labrador retriever with abnormal spinal column. Vet. Pathol., v. 14, n. 3, p. 256-260, 1977.

JEFERRY, N. D.; SMITH, P. M.; TALBOT, C. E. Imaging findings and surgical treatment of hemivertebrae in three dogs. J. Am. Vet. Med. Assoc., v. 230, n. 4, p. 532-536, 2007.

GRENN, H. H.; LINDO, D. E. Hemivertebrae with severe kypho-scolioses and accompanying deformities in a dog. Can. Vet. J., v. 10, n. 8, 1969.

MCMASTER, M J.; SINGH, H. Natural history of congenital kyphosis and kyphoscoliosis. J. Bone Joint Surg. Am., v. 81, n. 10, 1999.

MOISSONNIER, P.; GOSSOT, P.; SCOTTI, S. Thoracic Kyphosis Associated with Hemivertebra. Vet. Surg., v. 40, n. 8, p. 1029-1032, 2011.

SANTOS, T. C. C., VULCANO, L. C., MAMPRIM, M. J., MACHADO, V. M. V., Principais afecções da coluna vertebral em cães: estudo retrospectivo (1995-2005). Vet. Zootec., v. 13 , n. 2 , p. $144-152,2006$.

SCHEY, W. L. Vertebral malformations and associated somaticovisceral abnormalities Clin. Radiol., v. 27, n. 3, p. 341-353, 1976.

SCHLENSKER, E., DISTL, O. Prevalence, grading and genetics of hemivertebrae in dogs EJCAP, v. 23, n. 3, p.119-123, 2013.

TAYLOR, S. M. Parte nove: doenças neurosmusculares. In: NELSON, R. W.; COUTO, C. G. Medicina interna de pequenos animais. 4. ed. Rio de Janeiro: Elsevier, 2010.

WHEELER, J. L.; LEWIS, D. D.; CROSS, A. R.; SEREDA, C. W. Closed fluoroscopic-assisted spinal arch external skeletal fixation for the stabilization of vertebral column injuries in five dogs. Veterinary Surgery, v. 35, p. 442-448, 2007. 